\title{
Pizkunde: los «renacimientos» de la lengua vasca*
}

\author{
Piøkeunde: the «Revivals» of the Basque Language
}

\author{
Xabier ZabaltZa \\ xabier.zabaltza@ehu.eus \\ Universidad del País Vasco/Euskal Herriko Unibertsitatea \\ ORCID: orcid.org/0000-0001-8743-3084
}

\begin{abstract}
Resumen: En este trabajo se cuestionan el concepto y la cronología tradicional del Pizkunde, el «renacimiento» de la identidad vasca en la segunda mitad del siglo XIX. En realidad, antes de la aparición del nacionalismo, en los países vascos existieron dos «renacimientos» distintos, que solo tuvieron relación puntual entre ellos. El primero, de signo culturalista y en vascuence, fundamentalmente al norte de los Pirineos. El segundo, más político y fundamentalmente en castellano, al sur. El nacionalismo vasco procede mucho más de este que de aquel. Además, en la Vasconia meridional las reivindicaciones culturales son, en gran parte, consecuencia y no causa de las reivindicaciones políticas.
\end{abstract}

Palabras clave: lengua vasca, «renacimiento» literario, fuerismo, culturalismo, nacionalismo

Abstract: In this paper the concept and the traditional chronology of the Pizkunde, the «revival» of Basque identity in the second half of the 19th century, are questioned. In fact, before the emergence of nationalism, in the Basque countries there were two different «revivals», which only occasionally crossed paths. The first, which was culturalist and expressed in Basque, mostly to the north of the Pyrenees; the second, more political and mostly in Spanish, to the south. Basque nationalism has derived much more from the latter than from the former. In addition to this, in Southern Basqueland, cultural claims are largely a consequence and not a cause of political demands.

Keywords: Basque language, literary «revival», «fuerism», culturalism, nationalism.

\footnotetext{
* Este trabajo forma parte del Proyecto de Investigación MINECOG14/P11, financiado por el Ministerio de Economía y Competitividad. Una versión anterior del texto fue presentada como ponencia en el VII Seminari d'Estudis Catalans del Vuit-Cents, organizado por el Departamento de Filología Catalana de la Universitat de València, el 15 de diciembre de 2017. Agradezco al profesor Rafael Roca i Ricart su dedicación a la preparación del seminario y su apoyo en la redacción del artículo.
} 


\section{Introducción}

En la segunda mitad del siglo XIX se produjeron en los territorios del suroeste europeo con idioma propio distinto del estatal sendos «renacimientos» literarios. El Felibritge en Occitania, la Renaixença en los países de lengua catalana y el Rexurdimento en Galicia son conocidos y reconocidos tanto dentro como fuera de sus fronteras y han llegado a formar parte del bagaje de un español o francés culto medio. Por el contrario, pocas son las personas, incluso vascas, que han oído hablar del Pizkunde ${ }^{1}$, el movimiento análogo en Vasconia.

Si el propio concepto de «renacimiento» literario es discutible (Marfany 2004), de ahí las comillas que acompañarán al término a lo largo de todo el artículo, más todavía lo es el de Piøkeunde. Aquellos estudiosos que admiten su existencia suelen colocar sus límites temporales entre la abolición de los fueros de Álava, Guipúzcoa y Vizcaya en 1876 y el inicio de la Guerra Civil en 1936 (Torrealday 1977: 247-280). En este trabajo se cuestiona esta cronología, ya que la corriente iniciada en 1876 tuvo influencia sobre todo en la Vasconia española y, por lo tanto, deja al margen el movimiento anterior en la Vasconia francesa. De hecho, el «renacimiento» cispirenaico fue más identitario que literario, al menos en lo que a la lengua vasca respecta. De la misma manera que, como se verá, existen argumentos para negar la existencia del Pizkunde, también podría hablarse de dos o incluso tres pizkundes diferentes, ya que el culturalismo (euskaltraletasuna), especialmente vasco-francés, y el fuerismo (foruzaletasuna), casi exclusivamente vasco-español, con su continuación, el nacionalismo político (abertzaletasuna) tienen pocos puntos en común. A lo largo del artículo se explicará que los autores vascos de expresión castellana tienden a primar la peculiaridad jurídica sobre la lingüística, mientras que entre los de expresión euskérica (y francesa) ocurre lo contrario. Como sentenció el guipuzcoano Juan Ignacio de Iztueta (1767-1845), antes incluso de los primeros Juegos Florales, «Euscara ill ezquero Fueroac ez dira bicico; bañan Euscara bici bada, Fueroac piztuco dira. Fueroac nai dituanac, maite izan bear du Euscara» (Iztueta 1847: vi) ${ }^{2}$.

\section{Condicionamientos históricos}

El vascuence o euskara es un idioma no indoeuropeo, sin relación tipológica con familia lingüística conocida (Igartua y Zabaltza 2012). En España se habla en la totalidad de Guipúzcoa (Gipurkooa) y en parte de Vizcaya (Bižkaia), Navarra (Nafarroa) y Álava (Araba). En Francia, en las antiguas provincias

1 En vascuence, «renacimiento» se dice pizkeundea, siendo la «a» final el artículo. «Renacimiento Vasco», como suele ser denominado el movimiento en Vasconia, es Euskal Piøkundea. «Renacimientos», en plural, se dice piøkundeak. Dado que en castellano, lengua en la que está escrito este trabajo, sería redundante el uso de dos artículos (el pizkundea, los pizkundeak) he optado por dejar el término en el caso absoluto (pizkunde).

2 «Si muere el Vascuence los Fueros no vivirán; pero si vive el Vascuence, los Fueros renacerán [piztuco dira]. El que quiera Fueros, tiene que amar el Vascuence» (la traducción es nuestra). 
de Sola (Zuberoa), Baja Navarra (Baxenabarre) y Labort (Lapurdi), salvo en el área más septentrional de las dos últimas, de lengua tradicional gascona. Las capitales respectivas de estos siete territorios son San Sebastián (Donostia), Bilbao (Bilbo), Pamplona (Iruñea), Vitoria (Gasteiz), Mauleón (Maule), San Juan de Pie de Puerto (Donibane Garazı) y Bayona (Baiona). La Revolución abolió en 1789 las instituciones de las provincias vasco-francesas. Desde el año siguiente Labort, Baja Navarra y Sola ni siquiera existen administrativamente ya que fueron disueltas en el Departamento de los Bajos Pirineos (denominado desde 1969 «Pirineos Atlánticos»), en el que el Bearne, provincia no vasca, ocupa la mayor parte del territorio y de la población. Salvo un breve periodo durante la Guerra de la Independencia (1810-1813), Álava, Guipúzcoa y Vizcaya no habían formado nunca una unidad administrativa civil antes del período que nos ocupa y no volvieron a hacerlo, y solo de iure, hasta el Estatuto de Elgeta (1936-1937), que empezó a aplicarse cuando casi la totalidad de las dos primeras provincias estaba de facto en manos de los franquistas. Los escasos ocho meses en los que estuvo vigente fueron los únicos en la historia del euskara en los que fue reconocido como lengua cooficial antes de 1979. Por otro lado, Navarra fue un reino hasta que la Ley de Modificación de Fueros, llamada también «Paccionada», promulgada en 1841, tras la Primera Guerra Carlista, suprimió sus instituciones a cambio de una amplia autonomía en materia fiscal y administrativa, confirmada por el Convenio Económico, vigente desde 1877. Tras la Tercera Guerra Carlista, fueron abolidos los fueros de las tres provincias occidentales (1876), aunque los Conciertos Económicos, que deben no poco al precedente del Convenio navarro, vinieron a paliar en parte esa situación desde 1878. Resumiendo: dada la complejidad institucional de los territorios del euskara y la ausencia, hasta tiempos recientes, de una unidad política supraprovincial (aun parcial), más que de «País Vasco» en singular y con mayúsculas se debería hablar de «países vascos», en plural y con minúsculas (Zabaltza 2005). Más aún, «país», «vasco» y «país vasco» son tres galicismos, por lo que el nombre castizo en castellano para denominar al conjunto de los siete territorios en los que en mayor o menor medida se habla vascuence debería ser, en todo caso, «Vasconia» (Euskal Herria en euskara; Bascònia en catalán) (Fortuny 1906; Azaola 1972-1976: II, 15-43; Zabaltza 2005: 23-33).

Desde 1789, por lo menos, encontramos una diferencia sustancial entre la Vasconia española y la francesa. En Francia, además del Reino de la Baja Navarra y los Vizcondados de Labort y Sola, también Artois, Bearne, Borgoña, Bretaña, Cambrésis, Córcega, Flandes, Foix, Languedoc, Provenza y varios países gascones habían mantenido sus états hasta la Revolución, pero esta arrasó todo lo que olía a feudalismo. A partir de entonces en la parte septentrional de Vasconia no existirá un «hecho diferencial» institucional y ello explica que el vasquismo sea en sus tres entidades fundamentalmente lingüístico. Por el contrario, desde los decretos de Nueva Planta (1707-1716), que fulminaron las instituciones de la Corona de Aragón, el Reino de Navarra, los Condados de Vizcaya y Álava y la Provincia de Guipúzcoa fueron los únicos territorios de la Monarquía Hispánica que mantuvieron sus fueros. Esta es la razón por la que, contrariamente a su homónimo del norte, el vasquismo al sur de los Pirineos es fundamentalmente político.

A pesar de la idealización de la que han sido objeto, las instituciones vascas del Antiguo Régimen, 
surgidas en su mayoría en la Baja Edad Media, se basaban en la discriminación, social y lingüística. Durante décadas, acaso durante siglos, para ser apoderado de las Juntas Generales de Vizcaya hubo que saber leer y escribir en castellano, una lengua que, salvo en la ciudad de Orduña (Urduña), en algunas villas como Bilbao y en las Encartaciones (Enkartazioak), la comarca más occidental del señorío, solo hablaba una parte pequeña de la población, que además solía ser analfabeta. Hasta época muy tardía, y únicamente en Vizcaya y Guipúzcoa, el uso de la lengua vasca por parte de las instituciones forales sureñas fue mínimo. Por ejemplo, ni un solo documento de las Cortes de Navarra, que se reunieron hasta 1829, está redactado en euskara. Con las excepciones señaladas, en los órganos representativos se consideraba el conocimiento del castellano como un auténtico estigma de clase: solo los estamentos pudientes, educados en esa lengua, tienen acceso a ellas. En el norte, al parecer el Bilçar (Asamblea) de Labort utilizó la lengua vasca de modo más frecuente, lo cual puede estar relacionado con el hecho de que en la costa labortana existiera una incipiente burguesía vascófona y vascógrafa (Zabaltza 2013). Como punto de comparación, las Cortes de Cataluña, Valencia y las Mallorcas funcionaron en catalán hasta la Nueva Planta. Cuando los primeros catalanistas creen el concepto de Renaixença (por ejemplo, Rubió y Ors 1880) partirán de una concepción tripartita, típicamente judeocristiana, de Paraíso-Caída-Redención. Ese esquema tan simplista (hoy felizmente superado) no es aplicable ni siquiera como caricatura a los territorios de lengua vasca, donde la tradición literaria y administrativa es mucho más endeble que en los de lengua catalana. En Vasconia no hubo Paraíso y, por lo tanto, tampoco Caída. Solo Purgatorio o lo que es lo mismo: diglosia (Michelena 1977: 521).

En la segunda mitad del siglo XIX, cuando se producen los «renacimientos» culturalista y fuerista, los países vascos están en pleno proceso de transformación en los planos económico, político y demográfico, pero también lingüístico. En 1850 Vasconia tiene unos 860.000 habitantes, de los que casi el 20\% vive en las tres provincias norteñas, que han mantenido el euskara muy vivo. Si el Rosellón va durante la Renaixença a remolque de la Cataluña meridional, van a ser las provincias vasco-francesas, sobre todo Labort, las que lleven la iniciativa durante el primer piə̌kunde. Hasta el siglo XVIII la mayor parte de los escritores en euskara son de la Vasconia continental (Torrealdai 1997: 404-405) y no es casual que los primeros Juegos Florales se celebren aquí. En 1850 Navarra tiene casi 300.000 habitantes. Es, por mucho, la provincia más poblada y no solo eso: el 30 por ciento de los navarros hablan euskara. El Viejo Reino es el núcleo de una nacionalidad que no es consciente de serlo. Pero la lengua, con todo lo que conlleva, está moribunda en una amplia zona (desde entonces el vascuence ha perdido en Navarra la mitad de su territorio y, en proporción, dos tercios de sus hablantes). Pamplona, cuyo nombre euskérico, Iruñea, significa precisamente «la ciudad», es la más poblada de las capitales vascas, por encima de Bilbao, que no empieza su despegue hasta la industrialización del último cuarto del XIX. Entre las provincias vasco-españolas van a ser Guipúzcoa y Vizcaya, paradójicamente las que mayor inmigración foránea reciben, las que mejor conservan el euskara (en Álava los vascófonos andarían en torno al 10\% en 1850). 
Tabla 1. Población de Vasconia (c.1850-c.1900) (elaboración propia a partir de Azaola 1988: 40 y Erize Etxegarai 1997: 263).

$\begin{array}{llll} & 1855 / 1857 & 1900 / 1906 & \text { Vascófonos en 1863 } \\ \text { Álava } & 96.398(11,19 \%) & 96.385(8,87 \%) & 10 \% \\ \text { Guipúzcoa } & 156.493(18,17 \%) & 195.850(18,03 \%) & 96 \% \\ \text { Navarra } & 297.422(34,54 \%) & 307.669(28,33 \%) & 30 \% \\ \text { Vizcaya } & 160.579(18,65 \%) & 311.361(28,67 \%) & 81 \% \\ \text { Labort-Baja Navarra-Sola } & 150.000(17,42 \%) & 174.515(16,06 \%) & 65 \% \\ \text { Vasconia } & & & \end{array}$

\section{Preludio: Augustin Chaho}

Antes de internarnos en el estudio de los diferentes pizkeundes dedicaremos unas líneas a Augustin Chaho (Agosti Xaho, 1811-1858), el más importante precursor del nacionalismo vasco (Zabalo 1999; Zabaltza 2011). Escritor romántico, nacido en la provincia transpirenaica de Sola, en su juventud residió en París, donde estuvo en contacto con algunos de los mejores escritores franceses de su tiempo, como Charles Nodier, Théophile Gautier, Flora Tristan y, probablemente, el mismo Victor Hugo.

Como vasco, Chaho era continuador de los llamados «apologistas del vascuence» de los siglos XVI a XIX (Madariaga Orbea 2008), que, casi siempre en castellano, se esforzaron en demostrar que el euskara fue la primera lengua de las Españas e incluso, los más radicales entre ellos, también la del Paraíso terrenal. Como francés, combinó estas ideas con las de Court de Gébelin, quien, por medio de la lingüística, pretendió reconstruir la religión primitiva de la Humanidad. Chaho se presentaba como el profeta (ą̧tia) de un culto deísta de su invención en el que el pueblo vasco era el verdadero pueblo elegido y el euskara, su idioma sagrado. Por muy extravagantes que puedan resultar sus ideas hoy en día, Chaho contribuyó como pocos a la dignificación de la cultura y lengua vascas. No cabe duda de que el vidente suletino supuso un revulsivo mucho más impactante para la posterior evolución del «renacimiento» vasco que su sobrevalorado contemporáneo Aribau para el catalán (Zabaltza 2012).

La ideología de Chaho es compleja. En Francia se proclamaba republicano y así participó en la Revolución de 1848 en Bayona. En España simpatizaba con los carlistas y en 1835 fue testigo en Navarra del levantamiento en favor de don Carlos María Isidro, experiencia que narró en Voyage en Navarre pendant l'insurrection des Basques, donde planteó la primera carlistada como una guerra de liberación nacional vasca (Chaho 1865), un mito que conocería un éxito notorio en ETA y en la denominada izquierda abertzale. Para Chaho los fueros son la auténtica Constitución del pueblo 
vasco, idea poco recurrente en los autores norteños. Como tantos fueristas, antes y después que él, pensaba que mientras han estado vigentes, es decir, desde la noche de los tiempos, Vasconia ha sido independiente, a pesar de que celtas, fenicios, romanos, germanos, musulmanes y castellanos han provocado guerras sin cuento para acabar con su prístina libertad.

Chaho escribió la mayor parte de su obra en francés, pero su aportación a la etnología vasca no es desdeñable. Recogió un centenar de canciones populares (Chaho 2006) y dejó inconcluso un diccionario cuatrilingüe (vasco, francés, castellano y latín) (Chaho 1856). En 1848 publicó en Bayona el primer intento de un semanario en lengua vasca (Uscal-Herrico Gaseta) (Arkotxa 1998; Díaz Noci 2012: 84-86). En ocasiones mezcló tradiciones auténticas con productos de su imaginación, como la indocumentada covada vasca o Aitor y Amagoia, los patriarcas del pueblo euskaro. Propugnó una ortografía que superara el caos existente en el euskara de su época y optó, más en teoría que en la práctica, por el dialecto labortano, que no era el suyo, para crear una literatura común. En la dedicatoria de sus Études grammaticales sur la langue euskarienne, que escribió junto a Antoine d'Abbadie, encontramos una de las primeras alusiones al lema Zazpiak Bat, símbolo de la unidad de las siete provincias vascas de ambas vertientes del Pirineo (Abbadie y Chaho de Navarre 1836), que, ya de modo explícito, se difundiría desde los Juegos Florales de 1892.

Chaho solo demandaba la autonomía para las provincias vascas del sur. Incluso en algún momento parece que lo que pretendía en realidad era anexionarlas a Francia (Zabaltza 2005: 232-233). Nunca reivindicó el restablecimiento de los fueros labortanos, bajonavarros y suletinos abolidos en 1789 . En 1848 propuso la creación de un nuevo departamento, Adur y Gaves, con Bayona como capital, que no era propiamente una institución vasca, ya que habría incluido, además de las tres provincias de la Vasconia francesa, la subprefectura gascona de Dax (Arkotxa 1998: 134; Zabalo 1999: 159). A pesar de lo dicho, es cierto, como hemos escrito en algún otro lugar, que «Chaho era más político que los vasquistas de la Vasconia septentrional y más culturalista que los fueristas (y los nacionalistas) de la Vasconia meridional» (Zabaltza 2011: 49). En ese sentido, puede ser reivindicado como un precedente, tanto por la tradición vasco-francesa como por la vasco-española.

\section{Primer acto: el pizkunde culturalista (desde 1851)}

El «renacimiento» vasco-francés, basado en la lengua, suele ser obviado por los historiadores del nacionalismo vasco, que a menudo desconocen el euskara. Como Labort, Baja Navarra y Sola habían perdido sus instituciones al inicio de la Revolución y el piæ̌kunde septentrional no tiene interés en recuperarlas ni en crear una entidad territorial vasca, sería más correcto tildar este movimiento de «culturalista» que de «regionalista» (Zabaltza 2018). Sin embargo, precisamente debido a la ausencia de fueros, el vasquismo del norte, contrariamente al del sur, subsume por completo lo provincial (no hay labortanismo, ni bajonavarrismo, ni siquiera suletinismo, como sí hay navarrismo, vizcaínismo, 
alavesismo e incluso guipuzcoanismo). Su figura clave es Antoine d'Abbadie d'Arrast (Anton Abadia-Ürrüstoi, 1810-1897), descubridor, científico y antiguo colaborador de Chaho reconvertido en mecenas. Desde 1851 organizó en Labort los Juegos Florales en lengua vasca. Ese es el motivo por el que hemos colocado el origen del pi₹̌kunde culturalista en esa fecha, ya que, como ocurrió en los territorios de lengua catalana, gracias a ellos la lengua privativa adquirió una innegable presencia en la esfera pública. En euskara los juegos de d'Abbadie son denominados Koplarien Guduak, esto es, «Combates de Poetas». En ellos compitieron labradores, artesanos, médicos, sacerdotes e incluso militares (Michelena 1960: 172) como el labortano Jean-Pierre Duvoisin (1810-1891), traductor de la Biblia al vascuence (1859-1865). Por decirlo a la valenciana, en los Koplarien Guduak participaron por igual poetes de guant y poetes d'espardenya, aunque el modelo literario utilizado, basado en el versolarismo ${ }^{3}$, es más bien propio de la tradición oral. En 1877 los juegos tuvieron lugar en SaintPalais (Donapaleu, Baja Navarra), fuera ya de Labort, y en 1879, con la colaboración de la Asociación Euskara, cruzan por primera vez la frontera franco-española y se celebran en Elizondo (Navarra). Desde ahí se irán expandiendo a Sola (1880), Guipúzcoa (1881) y Vizcaya (1883). En Álava tendrán lugar en 1899, muerto ya d'Abbadie. Pese a su clara visión pan-vasca, la labor del mecenas suletino se ciñó casi siempre a la Vasconia transpirenaica: de los 49 Juegos Florales organizados a cuenta suya entre 1851 y 1899, 39 se celebraron en las tres provincias francesas (Tabla 2). Al norte de los Pirineos los Koplarien Guduak, como las Fiestas Vascas (Euskal Bestak) de los republicanos, eran exclusivamente en vascuence y perduraron más allá de la Primera Guerra Mundial. Al sur, además de los financiados por d'Abbadie, se prodigaron entre 1879 y 1936 otros certámenes, que, con la excepción de los del Ayuntamiento de San Sebastián (Euskarąko Hitz. Jostaldiak), solían combinar el vascuence y el castellano, incluso en zonas con un alto porcentaje de vascófonos monolingües (Dávila Balsera y Eizaguirre Sagardia 1995; Toledo Lezeta 1998).

Tabla 2. Sedes de los Juegos Florales organizados por d’Abbadie (hasta 1899) (elaboración propia a partir de Dávila Balsera y Eizaguirre Sagardia 1995: 269 y 295 y Urkizu 1997: 48-59).

Labort: Urrugne/Urruña (1851-1860, 1862 y 1863), Sare/Sara (1864-1869, 1871-1876, 1878, 1882, 1884 y 1885), Ascain/Azkaine (1887), Cambo-les-Bains/Kanbo (1888), Saint-Jean-de-Luz/Donibane Lohizune (1892, 1894 y 1897), Saint-Pée-sur-Nivelle/Senpere (1898). En total: 34.

Baja Navarra: Saint-Palais/Donapaleu (1877), Saint-Jean-Pied-de-Port/Donibane Garazi (1894). En total: 2.

Navarra: Elizondo (1879), Santesteban/Doneztebe (1889), Vera/Bera (1895). En total: 3.

Sola: Mauléon/Maule (1880, 1890 y 1896). En total: 3.

Guipúzcoa: Irún/Irun (1881), Urnieta (1886), Azpeitia (1893). En total: 3.

Vizcaya: Marquina/Markina (1883), Yurreta/Iurreta (1891), Villaro/Areatza (1897). En total: 3.

Álava: Aramayona/Aramaio (1899). En total: 1.

3 El versolarismo (bertsolaritza) es una técnica de improvisación de rimas, típica de los territorios vascos. 
Varias son las diferencias entre los Koplarien Guduak y los Jocs Florals. Nos detendremos en las más obvias. Para empezar, frente a Bofarull, Milà i Fontanals, Rubió i Ors y compañía, d'Abbadie no tenía conciencia de estar «restaurando» nada. Ni por su cantidad ni por su calidad es comparable la tradición literaria escrita en lengua vasca con la catalana. Por solo dar un dato, de los 723 libros publicados en vascuence entre 1545 y 1875, 639 (88,4\%) tienen una finalidad religiosa y solo 29 (4\%) pueden ser considerados como literarios (elaboración propia a partir de Torrealdai 1997: 57). Es posible, además, que d'Abbadie desconociera la existencia de certámenes poéticos en euskara en el siglo XVII, que le podrían haber servido de precedente. Por si fuera poco, como se ha visto, las instituciones del Antiguo Régimen funcionaban generalmente en castellano o en francés (o incluso en gascón), por lo que no había un glorioso pasado administrativo al que apelar.

Pero la diferencia fundamental de los Koplarien Guduak respecto a los Jocs Florals es su marcado carácter rural. Es cierto que también muchos jocfloralescos anhelaban el periodo anterior a la industrialización. Pero bastará un dato para corroborar esta afirmación. A mediados del siglo XIX, las sedes de los principales juegos en catalán, Barcelona y Valencia, tenían respectivamente 220.000 y 90.000 habitantes. Urruña, sede de los juegos en lengua vasca entre 1851 y 1863, apenas contaba con 3.600 habitantes. Por su parte, Sara, que, con interrupciones, sería su sustituta hasta 1885, andaba en torno a los 2.000 habitantes. Es más, los Koplarien Guduak evitan a propósito las grandes ciudades (incluyendo cinco de las siete capitales), que ya por entonces estaban muy castellanizadas o francesizadas.

Los juegos de d'Abbadie contribuyeron a difundir la nueva ortografía euskérica que intentaba abrirse camino gracias a los esfuerzos del grupo en torno al príncipe Louis-Lucien Bonaparte (1813-1891), vascólogo y vascófilo, sobrino de Napoleón. En una carta escrita a este en 1869 (en francés, naturalmente), su colaborador Duvoisin constata que «la nouvelle orthographe, popularisée par les chants de nos poètes, commence à être employée par la classe des paysans qui savent écrire; j'ai entre les mains plusieurs lettres dans lesquelles le zr remplit sa fonction, à l'exclusion du ç, où les qui et les gui ont entièrement disparu» (apud Zuazo Zelaieta 1988: 244). Cabe señalar que la prioridad, casi la obsesión, de Bonaparte era la dialectología, no la literatura, ni el uso administrativo del idioma, por lo que no se preocupó por la creación de una variante supradialectal.

D'Abbadie no era un nacionalista vasco. Su ideología estaba cerca del bonapartismo. En los Koplarien Guduak no se cuestiona la unidad de España y, menos aún, la de Francia. Tampoco el estado diglósico del euskara. El suletino es un claro ejemplo de «doble patriotismo» (Fradera 2003: 59-155; Rubio Pobes 2003: 153-177) pues tampoco puede dudarse de su profundo vasquismo. Ya se ha dicho que desde 1879 los Koplarien Guduak se celebraron también en la Vasconia española, por lo que se produjo cierta ósmosis entre el culturalismo del norte y el fuerismo del sur. Así algunos autores meridionales llegan a conceder al euskara la primacía absoluta entre los fundamentos de la nacionalidad vasca, por encima incluso de las «leyes viejas». En la línea de Iztueta, Felipe Arrese Beitia (1841-1906), poeta vizcaíno que resultó premiado en los juegos de Elizondo, uno de los 
contadísimos koplaris que se acercaría al nacionalismo, afirma: «Baldin euskera bizitzen ezpada/ iltzat daukadaz foruak» (Arrese Beitia 1879: 293). Si bien los Koplarien Guduak no se interesaron por el establecimiento de una lengua literaria y administrativa común que pudiera competir con el castellano y el francés, contribuyeron, en cambio, de modo determinante al fortalecimiento de una conciencia vasca por encima de los límites estatales. Desde los juegos de 1892 en San Juan de Luz (Donibane Lobizune, Labort) se difundió el lema Zarpiak Bat («las siete son una»), muy popular hoy en día, que simboliza la unidad de los cuatro territorios vascos de España y los tres de Francia (Altzibar 1998). Solo un año más tarde, en los juegos de Azpeitia (Guipúzcoa), en 1893, el labortano Gratien Adema «Zaldubi» (1828-1907), un conocido clérigo antirrepublicano, canta a la unidad de las siete provincias de Vasconia en unos versos que han pasado a la posterioridad, en los que no solo no niega, sino que explícitamente afirma la integridad territorial de España y Francia:

\author{
Zazpi Eskual herriek bat egin dezagun, \\ Guziak bethi, bethi, gauden gu Eskualdun. \\ I. Agur eta ohore Eskual herriari, \\ Laphurdi, Basa-Nabar, Zibero gainari, \\ Bizkai, Nabar, Gipuzko eta Alabari, \\ Zazpiak bat besarka loth beitez elgarri. \\ II. Haritz eder bat bada gure mendietan, \\ Zazpi adarrez dena zabaltzen airetan, \\ Frantzian, Espainian, bi alderdietan, \\ Hemen hiru 'ta han lau bat da zazpietan (Adema 1893: 388) ${ }^{5}$.
}

La perspectiva del culturalismo, que desde 1879 abarca en teoría el conjunto de los territorios vascos (aunque en la práctica excluye las comarcas de lenguas castellana, francesa y gascona), supera con creces la del fuerismo, centrado en la provincia. No hará falta recalcar que la unidad a la que apelaban «Zaldubi» y los demás koplaris no se entendía en términos políticos, por lo que no suponía ninguna amenaza para la identidad de los vascos que se sentían españoles y franceses.

No tiene sentido referirse en este artículo a todos los agentes del movimiento cultural euskérico de la segunda mitad del XIX. Pero resulta obligado dedicar al menos un párrafo a José María Iparraguirre (o Iparragirre, 1820-1881), por su influencia en el proceso de nacionalización vasca más allá de los límites provinciales. El Bardo, antiguo combatientes carlista que pasó gran parte del período entre 1839 y 1851 exiliado en Francia, incluida la Vasconia norteña, y que, según

\footnotetext{
$4 \ll \mathrm{Si}$ el vascuence no vive/ tengo a los fueros por muertos» (la traducción es nuestra).

5 «Unámonos los siete países vascos [Eskual herriek],/ Sigamos siendo todos vascos para siempre./ Gloria a Vasconia [Eskual herriari],/ a Labort, a la Baja Navarra, a Sola la alta,/ a Vizcaya, a Navarra, a Guipúzcoa y a Álava,/ Abracémonos las siete./ En nuestros montes hay un hermoso roble,/ que se abre con sus siete ramas,/ en Francia, en España, en los dos lados./ Aquí tres y allí cuatro, las siete son una sola» (la traducción es nuestra).
} 
confesión propia, no supo qué eran los fueros hasta bien cumplidos los treinta años (Mañé y Flaquer 1878-1880: II, 359), participó en los Juegos Florales de 1876 (en Sara, Labort) y 1879 (en Elizondo). A pesar de ser guipuzcoano, en 1852 o 1853 compuso el Gernikako Arbola, que convirtió el roble bajo el cual se reunían las Juntas Generales del Señorío de Vizcaya en un símbolo de todos los vascos («Zaldubi» alude a él en el pasaje citado). El Gernikako Arbola, a cuyo éxito contribuyeron ciertamente los Juegos Florales, pues su música se utilizaba para acompañar otras composiciones, es denominado en la prensa de la época «himno nacional» vasco. Ya en vida de su autor fue cantado con furor en las siete provincias, del Adur al Ebro, incluso en las comarcas de lengua castellana (Zabaltza 2017). Iparraguirre, que se sentía profundamente español, es otro caso de «doble patriotismo» que prueba que, antes de Sabino Arana, la nación vasca no se concebía como opuesta a la española ni a la francesa.

Más o menos heredera del culturalismo de d'Abbadie, en 1902 se constituyó Eskualz̧aleen Biltrarra, una asociación de amplio espectro ideológico, la primera que pretendió funcionar en las siete provincias, aunque, por razones que se explicarán, enseguida quedó limitada a las tres del norte. La principal publicación del vasquismo septentrional será Eskualduna, un seminario monolingüe en vascuence que aparecerá en Bayona entre 1887 y 1944 (Díaz Noci 2012: 120-121). Será un periódico muy conservador, nacionalista francés, hasta el punto que será clausurado a finales de la Segunda Guerra Mundial bajo la acusación de simpatizar con los nazis. Debe quedar claro que, junto a ese sector derechista y confesional (xuriak o «blancos», en el lenguaje de la época), en el vasquismo norteño existía un sector republicano (gorriak o «rojos») muy activo, representado por ejemplo, además de por Chaho, por dos labortanos que participaron en los Juegos Florales: JeanBaptiste Elissamburu (Joan Batista Elizanburu, 1828-1891), también militar, autor de Piarres Adame (1888), una de las primeras novelas en euskara, y Martin Guilbeau (1839-1912), alcalde de San Juan de Luz y primer presidente de Eskualzaleen Biltzarra. Ya se ha aludido al hecho de que los republicanos organizaron desde 1893 las Euskal Bestak, con la intención de hacer la competencia a los Koplarien Guduak de d'Abbadie. Habría que añadir que publicaron su propio seminario, Le Réveil Basque, bilingüe vascuence-francés, en Pau, capital del Bearne y del Departamento de los Bajos Pirineos, entre 1886 y 1895 (Díaz Noci 2012: 117-119).

\section{Segundo acto: el pizkunde fuerista (desde 1876)}

Desde el último decreto de Nueva Planta (1716), los territorios vascos fueron los únicos de la Monarquía de España que mantuvieron su especificidad institucional, por lo que los fueros se convirtieron en un elemento indisoluble de la identidad vasco-española. No es de extrañar que, según la historiografía tradicional, el Piøkeunde comience precisamente con la reacción popular que siguió a la abolición de las instituciones alavesas, guipuzcoanas y vizcaínas en 1876 (Navarra había 
seguido su propio camino en 1841, pero participó como la que más en el movimiento). En efecto, desde esa fecha se produjo en las cuatro provincias del sur un fuerte sentimiento de solidaridad intravasca y de rechazo al centralismo. Por entonces, se incrementó la literatura pseudohistórica y nostálgica de justificación del «hecho diferencial» perdido: autores como Francisco Navarro Villoslada (1818-1895), Antonio Trueba (1819-1889) o Vicente Arana (1847-1890), en los que el dominio de la lengua privativa suele brillar por su ausencia (Juaristi 1987). Es el momento también de las publicaciones periódicas que apoyan la causa fuerista. Por no perdernos en un maremágnum de nombres y fechas, solo citaremos una por cada provincia: Revista de las Provincias Euskaras (Vitoria, 1878-1880), Revista Euskara (Pamplona, 1878-1883), Euskal-Erria (San Sebastián, 18801918) y Revista de Viqcaya (Bilbao, 1885-1889) (Rubio Pobes 2003: 267-275; Díaz Noci 2012: 113115). El uso de euskara y derivados en los títulos de tres de estas revistas no debe inducir a engaño. A diferencia de Eskualduna e incluso Le Réveil Basque, las cuatro son publicaciones en castellano, aunque en todas ellas, en mayor o menor medida, tiene cabida la lengua vasca. Las principales organizaciones del movimiento fuerista, estructurado en torno a estas revistas, fueron la Asociación Euskara de Navarra (Nafarroako Euskarazko Elkargoa, 1877-1897), cuya figura más emblemática es Arturo Campión (1854-1937), y la Sociedad Euskalerria, de Bilbao (1880-1902), liderada por Fidel de Sagarmínaga (1830-1894). Entre los «euskalerriacos» vizcaínos y los «euskaros» navarros tuvieron una presencia significativa los liberales moderados que controlaron las instituciones forales hasta 1876 y no se integraron en la alta burguesía industrial y financiera (Elorza 1978: 11-107; Corcuera Atienza 2001: 119-176). Con todo, el fuerismo, como el culturalismo o el nacionalismo, es una ideología trasversal que incorpora elementos de diferentes procedencias.

Es indudable de que este movimiento fue capaz de impregnar de vasquismo los territorios meridionales y por eso tendría sentido colocar el inicio del Piqkunde en 1876, como se ha solido hacer. Hay que tener en cuenta, sin embargo, que la mayor parte de la literatura y de la publicística de reivindicación de las instituciones del Antiguo Régimen se produjo en castellano, que había sido la lengua principal y casi única de Cortes, Juntas Generales y Diputaciones Forales. El euskara aparece a menudo como otro «hecho diferencial» de la nacionalidad vasca, no como un medio de comunicación y, menos aún, como objeto de cultivo literario culto. Ayuda a entender esto saber que, para 1876, el idioma más usual en Pamplona, Vitoria y Bilbao es el español, que además es un signo de distinción de las clases medias y pudientes en las zonas vascófonas de las provincias del sur, incluyendo San Sebastián. Así mismo, cada uno de los cuatro territorios tuvo sus propios fueros durante el Antiguo Régimen, por lo que el vasquismo meridional, muy teñido de fuerismo, tendía a ser más provincialista que regionalista. Por ejemplo, Sagarmínaga, quien no parece conocer el vascuence, embriagado de furor historicista llega a hablar de «nación vizcaína», no de nación «vascongada» o «vasco-navarra» (Sagarmínaga 1880: xxviii-xxix) y eso que denominó Euskalerria a su sociedad. Como mucho, los fueristas aspiraban a una laxa confederación entre los cuatro territorios vascos peninsulares, un proyecto que muy pocas veces se explicita. Hay excepciones a ese provincialismo, por supuesto. Una de las más notorias es Arturo Campión, quien aprendió vascuence de adulto y fue autor, entre otras obras, de una gramática de sus cuatro dialectos 
literarios: vizcaíno, guipuzcoano, labortano y suletino (Campión 1884). El polígrafo pamplonés, a la vez navarrista y vasquista, superó los estrechos límites del fuerismo. En su obra, en la que se deja sentir la impronta tanto del romanticismo europeo como, específicamente, del culturalismo vascofrancés, plasmó una visión nacional vasca, a pesar de estar escrita prácticamente toda en su lengua materna, el castellano (Zabaltza 2005: 243-263).

Por las razones que se ha intentado explicar, el fuerismo es un movimiento casi al cien por cien cispirenaico. Es cierto, sin embargo, como ya se ha insinuado, que algunos vasco-españoles, normalmente vascófonos, eran sabedores de la corriente culturalista vasco-francesa, especialmente desde los Juegos Florales de 1879, los primeros que tuvieron lugar a este lado de la frontera. Ya conocemos a varios de esos autores: el propio Campión y koplaris como Arrese Beitia e Iparraguirre, quien antes de esa fecha ya había participado en los juegos. Otro de ellos fue el político neocatólico alavés Ramón Ortiz de Zárate (1817-1883), quien en 1867, es decir, también antes de los juegos de Elizondo, elaboró un proyecto de confederación euskara, en la que, algo novedoso, podrían participar las provincias vasco-francesas (Estornés Zubizarreta 1990: 56). Cabría mencionar asimismo al guipuzcoano José Manterola (1849-1884), de ideología liberal, director de la revista Euskal-Erria y principal promotor de los Juegos Florales (Euskarąko Hitz Jostaldiak) de San Sebastián. Y, por supuesto, al sacerdote vizcaíno Resurrección María de Azkue (1864-1951), que sería el primer presidente de la Academia de la Lengua Vasca (Euskaltzaindia) desde 1919. Azkue, vinculado durante un tiempo a la Sociedad Euskalerria de Bilbao, dirigió el que es tenido por el primer periódico vasco-español íntegramente en la lengua privativa: Euskalzale (Bilbao, 1897-1899) (Kintana Goiriena 2008: 394-399; Díaz Noci 2012: 148-150).

\section{Tercer acto: el pizkunde nacionalista (desde 1896)}

Hasta ahora hemos considerado el Pizkunde como una etapa previa al nacionalismo. Hemos afirmado que en realidad hubo dos pizkundes, uno en vascuence y otro en romance, con solo una interacción puntual entre ambos. En este apartado nos detendremos en el «renacimiento» que siguió al nacionalismo político, encarnado especialmente en su fundador, Sabino Arana.

Sabino Arana Goiri (1865-1903) fue un líder carismático que apareció en el momento de la caótica industrialización de Vizcaya a finales del siglo XIX. Su lengua materna era el castellano y, aunque llegó a aprender el vascuence de adulto como para escribir e incluso mantener una conversación sencilla, su conocimiento de la literatura euskérica era muy inferior al de Chaho, Campión o Azkue, por ejemplo. Arana provenía de una familia carlista, con la que tuvo que refugiarse en Bayona entre los años 1873 y 1876. La ciudad del Adur había estado separada de Labort entre 1177 y 1790 y en ella vivían pocos vascófonos, hasta el punto de que ni uno solo de los Koplarien Guduak tuvo lugar allí. De una cita retrospectiva de Sabino puede inferirse que el culturalismo de d'Abbadie no dejó 
huella en él durante su infancia bayonesa:

mi hermano y yo, con otros hijos de familias emigradas en Bayona solíamos sostener en el colegio de San Luis Gonzaga grandes pedreas contra lo que nosotros juzgábamos franceses y que, no obstante, eran tan vascos como nosotros, y tan lejos estaban de ser franceses como nosotros españoles (Arana Goiri 1901: 104).

Sorprendentemente, tampoco parece que el catalanismo ejerciera la menor influencia en Arana Goiri durante los cinco años que residió en Barcelona con la vana intención de estudiar Derecho (1883-1888). En cambio, del sacerdote integrista catalán Félix Sardá y Salvany (Fèlix Sardà i Salvany), partidario de que los católicos no se mezclaran con los no católicos, tomó la idea de separación entre vascos y españoles (Chacón Delgado 2012).

No sería inexacto considerar a Arana Goiri como un iconoclasta, ya que rompió con la tradición cuantas veces lo consideró conveniente. A él debemos un exitoso santoral pseudovasco (Miren, Kepa, Andoni, Jone...), producto íntegro de su imaginación, que vino a desterrar no solamente los nombres castellanos y franceses arraigados en el país (María/Marie, Pedro/Pierre, Antonio/Antoine, Juana/Jeanne...), sino también los auténticos nombres euskéricos (Mari, Peru/Piarres, Anton, Joana...) (Arana Goiri 1897a y 1898). Se opondrá asímismo a los Juegos Florales (Koplarien Guduak, Euskal Bestak, Euskarazko Hitz Jostaldiak, etcétera), que consideraba un carnaval extranjerizante (Arana Goiri 1897b). Tampoco era de su gusto el Gernikako Arbola de Iparraguirre e impondrá un himno nacional alternativo, el Eu₹ko-Abendearen Ereserrkija («Himno de la Raza Vasca»), más conocido como Gora ta Gora (Arana Goiri 1902), que hoy es oficial en la Comunidad Autónoma del País Vasco. Su voluntarismo palingenésico le llevó al punto de rechazar el término Euskal Herria, documentado desde 1567 (Pérez de Lazarraga 2004: 115-117) y que había sido utilizado por todos los fueristas y culturalistas de los siete territorios vascos. No puede ser casualidad que Eurkadi, denominación acuñada por Sabino con la confesa intención de remplazar tanto a Euskal Herria en vascuence como a «Euskaria» o «Euskeria» y a «Vasconia» en castellano, aparezca por primera vez el 21 de septiembre de 1896, en el escudo de la sociedad Renacimiento de la Historia y la Lengua de Bizkaya, que es también el acta de nacimiento del vocablo pižkunde (Arana Goiri 1896: 982). Para Sabino Arana el «renacimiento» vasco (en un principio, solo vizcaíno) no empieza con los Juegos Florales, ni tras la abolición foral, sino con el propio Sabino Arana. Pį̧kunde deriva de piz̨tu, «encenden». Es un neologismo bien formado que, conscientemente o inconscientemente, evita la idea de recuperación o renovación presente en su equivalente castellano («re-nacimiento»).

Pese a su iconoclasia, Sabino Arana no deja de ser un heredero, todo lo estridente que se quiera, del fuerismo provincialista vasco-español. Hay que recordar que se dio a conocer en 1892 con una obra titulada Bizkaya por su independencia y no, por ejemplo, Euskeria por su independencia (Arana Goiri 1892). Como Sagarmínaga y como su hermano mayor Luis Arana (1862-1951), al que atribuyó la pretendida revelación de la idea nacionalista (convincentemente negada por Chacón Delgado 2015), 
Sabino fue más vizcaínista que vasquista. El propio neologismo Euðkadi deriva de la inverosímil etimología sabiniana de «Vizcaya» (be-Eu₹ko-di-a, «conjunto de los vascos de abajo») (Zabaltza 1997). También el valor secundario otorgado a la lengua vasca en su cosmovisión indica que el nacionalismo político debe poco al culturalismo previo y, ya puestos, también al catalanismo o a cualquier otro movimiento afín. Arana Goiri no precisó de ningún influjo foráneo para proclamar lo que los autores fueristas llevaban décadas planteando en el plano teórico: el derecho a la secesión si la Corona violaba su pacto con los territorios vascos. Junto a la interpretación independentista de los fueros, la idea de «raza», la antigua «limpieza de sangre», es consubstancial a Sabino Arana, mucho más importante que el euskara. Como afirma en un artículo, significativamente titulado «Errores catalanistas»,

\begin{abstract}
No el hablar este ó el otro idioma, sino la diferencia del lenguaje es el gran medio de preservarnos del contagio de los españoles y evitar el cruzamiento de las dos razas. Si nuestros invasores aprendieran el Euskera, tendríamos que abandonar éste, archivando cuidadosamente su gramática y su diccionario, y dedicarnos á hablar el ruso, el noruego ó cualquier otro idioma desconocido para ellos, mientras estuviésemos sujetos a su dominio (Arana Goiri, 1894).
\end{abstract}

Para Sabino, la lengua no es un medio de comunicación, sino de separación. De los 312 artículos publicados por él en sus periódicos Bizkaitarra (1893-1895), Baserrittarra (1897), El Correo Vasco (1899) y La Patria (1901-1903), 57 están redactados en euskara, esto es, el 18 por ciento, un porcentaje muy inferior al de vascófonos vizcaínos de la época. La lengua vasca ocupa en el ideario de Arana Goiri un lugar bastante menos destacado que el integrismo católico, el racismo antiespañol, el independentismo o el historicismo fuerista. Hay que añadir que, por su purismo ultravizcaíno, los textos euskéricos sabinianos resultan prácticamente incomprensibles para los vascófonos de carne y hueso, por lo que, muy a menudo, van acompañados de su versión en castellano, que es casi siempre la original. Y aún así, al lado de la publicación socialista La Lucha de Clases, de Bilbao, cualquier cosa parece mucho, pues en esta no se documenta ni un solo artículo en euskara entre 1894 y 1901 (Larronde 1977: 192, 198, 246, 304 y 305, nota 192).

Sabino Arana fundó, en 1895, el denominado «Consejo Supremo Vizcaíno» (Bižkai-Buru-Batzarr en el peculiar idiolecto sabiniano) $)^{6}$, la primera de las ejecutivas provinciales del Partido Nacionalista Vasco (Euzko-Alderdi Jeltralia) ${ }^{7}$ (Corcuera Atienza 2001: 449-493). Todas las demás asambleas territoriales se establecerían después de su muerte: la guipuzcoana en 1908, la navarra y la alavesa en 1911 y la septentrional (en realidad, solo labortana) en 1990 (Pablo y Mees 2005: 38, 56 y 426).

6 Bizkai-Buru-Batzarr significa literalmente «Vizcay-Cabeza-Asamblea». «Consejo Supremo Vizcaíno», en euskara, es Bizkaiko Batzar Nagusia o, mejor incluso, Bizkaiko Kontseilu Nagusia.

7 Euzko, alderdi (en el sentido de «partido político») y jeltzale son tres neologismos sabinianos. Este último vocablo significa "partidario de JEL», acrónimo de Jaun-Goikua eta Lagi-Zarra, «Señor de lo Alto (es decir, Dios) y Ley Vieja (es decir, Fueros)». Por si fuera poco, lagi es otro neologismo para evitar el romanismo lege. En un vascuence menos esotérico, «Partido Nacionalista Vasco» se dice Euskal Herriko Partidu Nazionalista. 
El partido en ciernes aumentó su presencia social con la incorporación desde 1898 de antiguos miembros de la Sociedad Euskalerria, liderados por el empresario naviero Ramón de la Sota (1857-1936) (Corcuera Atienza 2001: 493-571). Los «euskalerriacos» conferirán al nacionalismo un elemento burgués, mucho más tendente al pragmatismo que al separatismo. Se ha hablado de Azkue y de su vasquismo lingüístico, pero, en general, los miembros de la Sociedad Euskalerria, que terminaría disolviéndose en 1902, todavía estaban más castellanizados que Arana Goiri y concedían más viabilidad al «dialecto bilbaíno» (un castellano salteado de palabras euskéricas) que al vascuence (Juaristi 1999). También en la Euskara de Navarra había pocos vascohablantes (Erize Etxegarai 1997: 519). Algún socio (el propio Campión) se incorporó al PNV, pero la asociación languidecía por el abandono de sus miembros liberales tras el fracaso del proyecto de Partido Fuerista en las elecciones provinciales de 1880 y desapareció definitivamente en 1897 (García-Sanz Marcotegui 2000). En cuanto a la otra asociación a la que nos hemos referido en este trabajo, la culturalista Eskualzaleen Biltzarra, que en un principio operaba en las siete provincias de Vasconia, la desidia de los sabinianos la redujo a los territorios del norte (Goyhenetche 1993), en los que pervive en nuestros días con más pena que gloria.

No es este el lugar para reescribir la historia del nacionalismo vasco, ni para negar las aportaciones de muchos nacionalistas a la cultura euskérica. Pero hay que insistir en que, para el nacionalismo, la lengua está supeditada a su proyecto político. Por decirlo con las palabras de su fundador,

Ezta ezebez euzkelduna ixatia, aberrtzalia ixan ezik.

Dana da aberrtzaletasuna, euzkerea jakin ezik be [...].

\section{$\mathrm{X}$}

Zabaldu egixu aberrtzaletasuna, ta berakin euzkerea be zabalduko da.

Zabaltzen ba-dozu euzkerea aberri bako izkeratzat, Aberrijaren arerijuak berakin zabalduko dira.

$\mathrm{X}$

Asko dira euzkerea eztakijen euzkotarrak. Au txarra da.

Batzuk dira euzkerea dakijen maketuak. Au txarragua da.

\section{$\mathrm{X}$}

Euzkerea eztakijen eun maketok kalte andija egitten dautsae geure Aberrijari.

Andijagua da, euzkerea dakijan maketo batek bakarrik, egitten dautsan kaltia [...].

Euzkerea eztakijan euzkotarr aberrtzaliak, ikasten dau.

Aberrtzalia eztan euzkeldun euzkotarrak, euzkerea aizten dau (Arana Goiri 1897c) ${ }^{8}$.

8 «No es nada ser euzkeldun [sic, vasco de lengua], sin ser patriota./ El patriotismo lo es todo, aunque no se sepa euzkera $[$ sic $][. .$.$] ./ Difunde el patriotismo, y con él también se expandirá el euzkera./ Si difundes el euzkera como una$ lengua sin patria, con él se expandirán los enemigos de la Patria./ Muchos son los euzkotarr [sic, vascos de «raza»] que no saben euzkera. Eso es malo./ Hay algunos maketos [término despectivo para referirse a los habitantes de otras zonas de España, catalanes incluidos] que saben euzkera. Eso es peor./ Cien maketos que no saben euzkera hacen mucho daño a nuestra Patria./ Mayor es el daño que le hace un solo maketo que sepa euzkera [...]./ El euzkotarr 
El euskara solo se convirtió en una prioridad para el nacionalismo de raigambre sabiniana con la dictadura de Primo de Rivera, que, al reprimir las manifestaciones políticas de la nacionalidad vasca, forzó a los militantes al activismo cultural, ya que este era tolerado por el régimen (Zabaltza 2005: 124-125). Como adujo el poeta guipuzcoano «Xabier Lizardi» (José María Aguirre o Agirre, 1896-1933), una de las cumbres literarias del tercer pizkunde, «Ezin genezakek abertzale bidetik jo. Euskeltzaletasunara [sic] jo dezagun» (apud Ariztimuño 1933: 456) 9 . Mutatis mutandis, en la segunda dictadura del siglo XX, mucho más brutal que la primera, se reproducirá la misma estrategia. Tal es así que ya para 1955 se recuperó la media anual de libros publicados en euskara antes de la Guerra Civil: 30 (Torrealdai 1997: 115 y 134).

\section{Colofón}

El historiador checo Miroslav Hroch es conocido por su tipología trifásica de los «movimientos nacionales» de los «grupos étnicos no dominantes». Según él (Hroch 1968), a la Fase A, de «interés académico», previa al nacionalismo político, seguiría la Fase B de «agitación patriótica» y a esta, la Fase $\mathrm{C}$ o «movimiento de masas». La Fase A coincide con lo que en Vasconia se denomina Piqkunde. Pero... ¿qué pizkunde? Sabino Arana, con quien indudablemente comienza la Fase B, es continuador de la tradición fuerista en castellano, no de la culturalista en vascuence. Recuérdese que Arana Goiri abominó de los Juegos Florales (postura que modificó desde 1898, con el ingreso de los antiguos «euskalerriacos» en el PNV) (Corcuera Atienza 2001: 516). La diferencia con el caso catalán es palmaria, ya que Prat de la Riba (1898: 610 y 1906: 124), por citar una figura señera de las fases B y C en Cataluña, siempre consideró a los Jocs Florals y en general a la Renaixença, como un precedente del nacionalismo.

Pero las diferencias van más allá. Como puede comprobarse en la Tabla 3, la repercusión de los «renacimientos» culturalista y fuerista en la producción bibliográfica en lengua vasca fue mínima. Es más, el número de libros en vascuence aumenta, y de modo muy discreto, solo después de la aparición del nacionalismo político. Según este criterio, en Vasconia o, al menos, en su vertiente española, las reivindicaciones culturales son, en gran medida, posteriores a las reivindicaciones políticas y no la inversa. Contrariamente al esquema de Hroch, la Fase A es posterior a la Fase B e incluso a la Fase C, que, en Vizcaya y Guipúzcoa, puede considerarse consolidada para la proclamación de la Segunda República en 1931. En este sentido, la aportación de Sabino Arana es determinante,

patriota que no sabe euzkera, lo aprende./ El euzkotarr euzkeldun que no es patriota olvida el euzkera» (la traducción es nuestra). Este texto es una buena muestra del vascuence sabiniano, muy difícil de entender salvo para los iniciados. En unas pocas líneas se abarrotan tres neologismos (aberrtzale, aberrtzaletasun y eu₹kotarr), un leonesismo (maketo) y la ortografía etimologista, pero incorrecta, del grupo eusk-.

9 «No podemos seguir la vía del nacionalismo. Sigamos la del culturalismo» (la traducción es nuestra). 
a pesar de que escribiera sólo una mínima porción de su obra en vascuence y a pesar de que, por su purismo extremo, sus artículos y poemas en esta lengua sean inasequibles para un vascófono medio. A pesar de esos pesares y de varios más, se ha afirmado que «él sólo ganó para el euskera un número mayor de lectores que todos los que había tenido hasta entonces» (Escalera Maidagán apud Ibarzabal 1977: 26-27). A la postre, Sabino tenía bastante razón cuando, sin falsa modestia, se arrogó el mérito del «renacimiento» vasco. Pero las diferencias respecto al catalanismo, donde sí es válido el esquema de Hroch (Fase A: Juegos Florales, 1859; Fase B: Primer Congreso Catalanista, 1880; Fase C: Solidaritat Catalana, 1906), no terminan aquí. Las más importantes, ya se han mencionado, fueron el racismo exacerbado y la supeditación del idioma al avance del nacionalismo. Eso ha significado que en Vasconia siempre se ha podido ser nacionalista, incluso radical, sin saber una palabra de la lengua considerada nacional. Algo impensable en Cataluña. Junto a estas diferencias manifiestas hay que citar también la opción de Sabino por los dialectos y su oposición a la creación de un idioma literario común (Arana Goiri 1896).

Los tres «renacimientos» estudiados en este trabajo compartían, cada uno en su medida, uno de los tópicos sobre la identidad vasca más asentados, cual es la atávica asociación entre el euskara y el caserío. No fue hasta los años 60 , en plena dictadura franquista, cuando en la parte española del país la nueva industrialización generó la aparición de una clase media, en parte de origen rural, que, rompiendo con una inercia de siglos, había mantenido el idioma. La lengua vasca se convirtió en un símbolo de resistencia antifascista. Tras un largo proceso (Zuazo Zelaieta, 1988), en 1968 (año de muchas connotaciones y, más aún, en los países vascos), con la oposición del sector más ortodoxo del nacionalismo, la Academia de la Lengua Vasca estableció una variante estándar del vascuence (euskara batua) que, pese a las polémicas que suelen acompañar a los hechos trascendentales, fue rápidamente aceptada en su forma escrita en las zonas vascófonas de las siete provincias y que incluso se ha intentado extender, también en su forma oral, a las no vascófonas. En los 60, en gran medida debido a la unificación del idioma, se dio un auténtico boom de la literatura euskérica, cuyos frutos seguimos recogiendo medio siglo después. En los años finales del franquismo se superó, por primera vez, el listón de cien libros anuales en lengua vasca. Fue entonces también cuando se realizó de verdad algo parecido a una síntesis entre las tradiciones vasco-francesa, culturalista, y vasco-española, más política. El bajonavarro Fernando Aire «Xalbadon» (1920-1976) fue capaz de expresarlo en verso mucho mejor que cualquier prosa:

\footnotetext{
7/ Anai-arrebak, entzun ene aho-otsa: izaite bat ez daike hezur hutsez osa; herria da gorputza, hizkuntza bihotza; bertzetik berextean bitarik bakotxa, izaite horrendako segurra hil hotza.

8/ Batzu herriaz orroit, euskaraz ahantzi; bertzek euskara maita, herria gaitzetsi;
} 
hizkuntza ta herria berex ez doatzi, berek nahi daukute konpreniarazi bata bertzea gabe ez daizkela bizi (Xalbador 1989: 180) ${ }^{10}$.

En los años 60 del siglo pasado el euskara se convirtió en el elemento definitorio de la nacionalidad vasca, dejando a un lado, por fin, el racismo y el confesionalismo impuestos por el fundador del nacionalismo. Podríamos incluso afirmar que los «renacimientos» de 1851, 1876 y 1896 fueron meros precedentes y que el verdadero Piðkunde, con mayúscula, empezó en 1968. Lo grotesco es que esa síntesis se produjo mientras el mundo tradicional vasco, el que añoraban por igual culturalistas, fueristas y nacionalistas, desaparecía para siempre y mientras los tres territorios del norte, en pleno proceso de terciarización y desprovistos de instituciones, se desnacionalizaban a pasos agigantados. No hará falta recordar que esa desnacionalización ha ocurrido (está ocurriendo) sin que mediara dictadura alguna.

El largo y difícil camino de la lengua vasca está lleno de paradojas. No es la menor que casi siempre haya sido un elemento secundario para el nacionalismo y que, a la vez, no pueda sobrevivir sin el nacionalismo. La reciente experiencia vasco-francesa, sobre todo labortana, parece demostrar que no basta el culturalismo para mantener una lengua minoritaria en una sociedad urbana y en un mundo cada vez más globalizado. En cuanto al fuerismo, hace tiempo ya que fue subsumido por el nacionalismo en las dos provincias meridionales más vascófonas (Vizcaya y Guipúzcoa, cuyo régimen fiscal fue derogado por el franquismo), mientras que en las dos más castellanizadas lingüísticamente (Álava y, sobre todo, Navarra, que mantuvieron el Convenio/Concierto durante la dictadura), ha incrementado en las últimas décadas su carácter aislacionista, convirtiéndose de hecho en una ideología antivasquista. Pero, como diría Kipling, esa es otra historia.

Tabla 3. Producción bibliográfica en euskara (1545-1994) (elaboración propia a partir de Torrealdai 1997: 68, 91, 115, $134-135$ y 176).

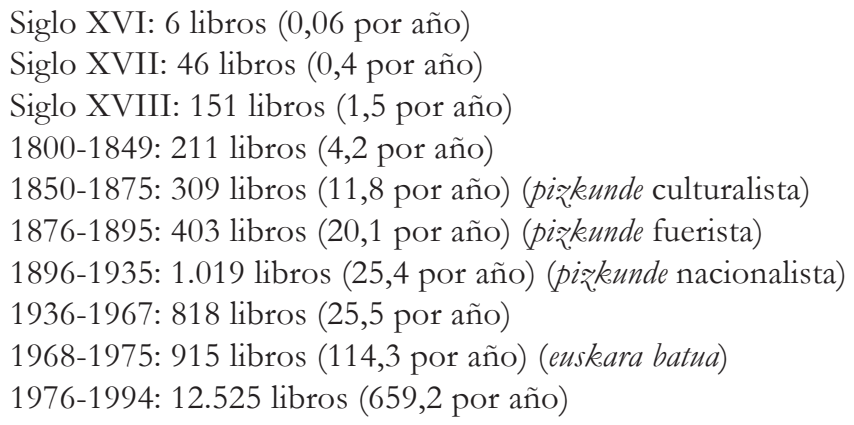

10 «Hermanos y hermanas, escuchad mi voz:/ no se puede crear una identidad solo con huesos; / la tierra es el cuerpo, la lengua es el corazón;/ si se separa la una de la otra,/ esa identidad, sin duda, muere./ Algunos piensan en el país, pero se olvidan del euskara;/ otros aman el euskara, pero desprecian el país;/ la lengua y la tierra no van separadas,/ ambas nos quieren hacer comprender/ que una no puede vivir sin la otra» (la traducción es nuestra). 


\section{Bibliografía}

Abbadie, A.Th. d' y Chaho de Navarre, J.A. (1836) Études grammaticales sur la langue euskarienne, Paris, Arthus Bertrand.

Adema, G. (1893) «Gauden eskualdun», en Urkizu, P. (ed.) (1997) Anton Abbadiaren koplarien guduak. Bertso eta aire zenbaiten bilduma (1851-1897), Bilbo/Donostia, Eusko Ikaskuntza/Euskaltzaindia, p. 388.

Altzibar, X. (1998) «'Zazpiak Bat’ gaia XIX. mendean», en Antoine d'Abbadie (1897-1997), Congrès International, Bilbo/Donostia, Eusko Ikaskuntza/Euskaltzaindia, pp. 663-688.

Arana Goiri, S. (1892) «Bizkaya por su independencia», en Arana Goiri, S. (1980) Obras completas, San Sebastián, Sendoa, vol. 1, pp. 107-153.

. (1894) «Errores catalanistas», Biøkaitarra, 31 de octubre de 1894.

—. (1896) «Lecciones de ortografía del euskera bizkaino», en Arana Goiri, S. (1980) Obras completas, San Sebastián, Sendoa, vol. 2, pp. 810-982.

—. (1897a) «Egutegi bizkattarra», en Arana Goiri, S. (1980) Obras completas, San Sebastián, Sendoa, vol. 2, pp. 983-1015.

—. (1897b) «Las Fiestas Euskaras», Baserrittarra, 9 de mayo de 1897.

—. (1897c) «Aberrija», Baserrittarra, 6 de junio de 1897.

. (1898) «Lenengo egutegi bizkattarra», en Arana Goiri, S. (1980) Obras completas, San Sebastián, Sendoa, vol. 2, pp. 1401-1659.

—. (1901) «Fragmento de interview», en Corcuera, J., et al. (ed.) (1991) Historia del nacionalismo vasco en sus documentos. Iconografía nacionalista, Bilbao, Eguzki, vol. 1, pp. 101-104.

- (1902) «Euzko-Abendearen Ereserrkija», en Arana Goiri, S. (1980) Obras completas, San Sebastián, Sendoa, vol. 3, p. 2413.

Ariztimuño, J. (1933) «El patriota, el poeta, el cristiano (Joxe Mari de Agirre, Xabier de Lizardi, ha muerto)», en Ariztimuño, J. (1986-1988) Obras completas, San Sebastián, Erein, V, pp. 456-458.

Arrese Beitia, F. (1879) «Ama euskeriari azken agurrak», en Urkizu, P. (ed.) (1997) Anton Abbadiaren koplarien guduak. Bertso eta aire zenbaiten bilduma (1851-1897), Bilbo/Donostia, Eusko Ikaskuntza/ Euskaltzaindia, pp. 293-294.

Arkotxa, F. (1998) «150e anniversaire de l'Uscal-Herrico Gaseta», Lapurdum, III, pp. 125-201.

Azaola, J.M. de (1972-1976) Vasconia y su destino, Madrid, Revista de Occidente (2 vol.). . (1988) Elpaís vasco, Madrid, Instituto de Estudios Económicos.

Campión, A. (1884) Gramática bascongada de los cuatro dialectos literarios de la lengua éuskara, Tolosa, E. López. 
Chacón Delgado, P. J. (2012) «Introducción al estudio de la etapa barcelonesa de Sabino Arana Goiri (1883-1888)», Letras de Deusto, 134, pp. 155-181.

- (2015) «El concepto de independencia vasca en Sabino Arana Goiri», Historia Contemporánea, 50 , pp. $75-103$.

Chaho, J. A. (1856) Dictionnaire Basque, Français, Espagnol et Latin, d'après les meilleurs auteurs classiques et les dictionnaires des Académies française et espagnole, Bayonne, Lespés Éditeur.

Éditeur.

Chaho, A. (2006) Agosti Chahoren kantutegia (1844-1855), Zarautz, Susa. Patri Urkizuren edizioa.

Corcuera Atienza, J. (2001) La patria de los vascos. Orígenes, ideología y organización del nacionalismo vasco (1976-1903), Madrid, Taurus.

Dávila Balsera, P., y Eizaguirre Sagardia, A. (1995) «Las Fiestas Euskaras en el País Vasco (18791936)», en Dávila Balsera, P. (coord.) Lengua, escuela y cultura. El proceso de alfabetización en Euskal Herria (siglos XIX y XX), Bilbao, Universidad del País Vasco, pp. 257-311.

Díaz Noci, J. (2012), Historia del periodismo vasco (1600-2010), San Sebastián, Sociedad de Estudios Vascos.

Eloræa, A. (1978) Ideologías del nacionalismo vasco (1876-1937). De los «euskaros» a Jagi-Jagi, San Sebastián, Luis Haranburu Editor.

Erize Etxegarai, X. (1997) Nafarroako euskararen bistoria soziolinguistikoa (1863-1936). Soziolinguistika bistorikoa eta bizkeuntza gutxituen bizitza, Iruñea, Nafarroako Gobernua.

Estornés Zubizarreta, I. (1990) La construcción de una nacionalidad vasca. El autonomismo de Eusko Ikaskuntra (1918-1931), San Sebastián, Sociedad de Estudios Vascos.

Fortuny, C. de (1906) Els catalans á Basconia. Crónica de un viatje, Barcelona, Lliga Regionalista de Barcelona.

Fradera, J. M. (2003) [1992] Cultura nacional en una sociedad dividida. Cataluña, 1828-1868, Madrid, Marcial Pons.

García-Sanz Marcotegui, Á. (2000) «Los liberales navarros ante la irrupción del euskarismo», en Jimeno, R. (coord.) El euskera en tiempos de los euskaros, Pamplona, Gobierno de Navarra/Ateneo Navarro, pp. 145-218.

Goyhenetche, J. (1993) «Les origines sociales et historiques de l'association Eskualzaleen Biltzarra (1893-1913)», Bulletin du Musée Basque, 135, pp. 1-68.

Hroch, M. (1968) Die Vorkämpfer dernationalen Bewegung bei den kleinen Völkern Europas. Eine vergleichende Analyse zur gesellschaftlichen Schichtung der patriotischen Gruppen, Praha, Universita Karlova.

Ibarzabal, E. (1977) Koldo Mitxelena, San Sebastián, Erein. 
Igartua, I., y Zabaltza, X. (2012) Breve historia de la lengua vasca, San Sebastián, Etxepare Euskal Institutua.

Iztueta, J. I. de (1847) Guipuzcoaco Provinciaren condaira edo bistoria ceñetan jarritzen diraden arguiro asieratic orain-arte dagozquion berri gogoangarriac, Donostia, Ignacio Ramón Baroja-ren moldizteguia.

Juaristi, J. (1987) El linaje de Aitor. La invención de la tradición vasca, Madrid, Taurus.

—. (1999) El «chimbo» expiatorio (La invención de la tradición bilbaina), Madrid, Espasa Calpe.

Kintana Goiriena, J. (2008) Intelektuala nazioa eraikitzen: R.M. Aqkueren pentsaera eta obra, Bilbo, Euskaltzaindia.

Larronde, J.C. (1977) El nacionalismo vasco. Su origen y su ideología en la obra de Sabino Arana-Goiri, San Sebastián, Txertoa.

Madariaga Orbea, J. (2008) Apologistas y detractores de la lengua vasca. San Sebastián, Fundación para el Estudio del Derecho Histórico y Autonómico de Vasconia.

Mañé y Flaquer, J. (1878-1880) El oasis: viaje al país de los Fueros, Barcelona, Imprenta de Jaime Jepús Roviralta (3 vol.).

Marfany, J. Ll. (2004) «Llengües sense estat i renaixences romàntiques», en Marfany, J.Ll. (2008) Llengua, nació i diglòssia, Barcelona, L’Avenç, pp. 273-301.

Michelena, L. (1960) «Historia de la literatura vasca», en Michelena, L. (2011) Obras completas, San Sebastián/Vitoria, Seminario de Filología Vasca «Julio de Urquijo»/Universidad del País Vasco, XIII, pp. 81-205.

- (1977) «El largo y difícil camino del euskara», en Michelena, L. (2011) Obras completas, San Sebastián/Vitoria, Seminario de Filología Vasca «Julio de Urquijo»/Universidad del País Vasco, X, pp. 501-521.

Pablo, S. de, y Mees, L. (2005) Elpéndulo patriótico. Historia del Partido Nacionalista Vasco (1895-2005), Barcelona, Crítica.

Pérez de Lazarraga, J. (2004, escrito en 1567) Dianea eta koplak, Donostia, Erein. Patri Urkizuren edizioa.

Prat de la Riba, E. (1898) «Compendi de la història de Catalunya», en Prat de la Riba, E. (2000) Obra completa, Barcelona, Institut d'Estudis Catalans/Proa, vol.1, pp. 572-610.

- (1906) «La nacionalitat catalana», en Prat de la Riba, E. (2000) Obra completa, Barcelona, Institut d'Estudis Catalans/Proa, vol. 3, pp. 117-170.

Rubio Pobes, C. (2003), La identidad vasca en el siglo XIX. Discurso y agentes sociales, Madrid, Biblioteca Nueva.

Rubió y Ors, J. (1880) «Breve reseña del actual renacimiento de la lengua y literatura catalanas», Memorias de la Academia de Buenas Letras de Barcelona, III, pp. 141-238. 
Sagarmínaga, F. (1880) Memorias históricas de Vižaya, Bilbao, Imp. Lit. y Lib. de Juan E. Delmas.

Toledo Lezeta, A.M. (1998) «Antoine d'Abbadie Hegoaldean (1879-1895)», en Antoine d'Abbadie (1897-1997), Congrès International, Bilbo/Donostia, Eusko Ikaskuntza/Euskaltzaindia, pp. 535546.

Torrealdai, J. M. (1997) Euskal kultura gaur. Liburuaren mundua, Oñati, Jakin.

Torrealday, J. M. (1977) Euskal idąleak, gaur. Historia social de la lengua y literatura vascas, Oñati, Jakin.

Urkizu, P. (ed.) (1997) Anton Abbadiaren koplarien guduak. Bertso eta aire zenbaiten bilduma (1851-1897), Bilbo/Donostia, Eusko Ikaskuntza/Euskaltzaindia.

Xalbador [=Aire, F.] (1989) Odolaren mintroa, Tolosa, Auspoa Liburutegia.

Zabalo, J. (1999) Augustin Chabo ou l'irrintzina du matin basque, Biarritz, Atlantica.

Zabaltza, X. (1997) «El significado oculto de la palabra 'Euzkadi’», Fontes Linguae Vasconum, 74, pp. $77-83$

—_. (2005) Mater Vasconia. Fueros, lenguas y discursos nacionales en los países vascos, San Sebastián, Hiria.

—. (2011) Augustin Chaho, precursor incomprendido (1811-1858), Vitoria, Gobierno Vasco.

—_. (2012) «Agosti Xaho eta Euskal Pizkundea», Jakin, 189, pp. 11-22.

—_. (2013) «De la lingua Navarrorum al Estado Vasco», Historia Contemporánea, 47, pp. 471-492.

—_. (2017) «Gernikako Arbola, un himno huérfano», Historia Contemporánea, 54, pp. 207-241.

- - (2018) «iDel «renacimiento» literario al nacionalismo político? Una comparación entre los territorios de lengua catalana y los de lengua vasca», Historia y Política, 39, pp. 141-170.

Zuazo Zelaieta, K. (1988) Euskararen batasuna, Bilbo, Euskaltzaindia. 